\title{
Color singlet suppression of quark-gluon plasma formation
}

\author{
Jes Madsen, Dan M. Jensen and Michael B. Christiansen \\ Institute of Physics and Astronomy, University of Aarhus, DK-8000 Arhus C, Denmark \\ (June 7, 1995)
}

\begin{abstract}
The rate of quark-gluon plasma droplet nucleation in superheated hadronic matter is calculated within the MIT bag model. The requirements of color singletness and (to less extent) fixed momentum suppress the nucleation rate by many orders of magnitude, making thermal nucleation of quark-gluon plasma droplets unlikely in ultrarelativistic heavy-ion collisions if the transition is first order and reasonably described by the bag model.
\end{abstract}

25.75.+r, 12.38.Mh, 12.39.Ba, 24.85.+p

To appear in PHYSICAL REVIEW C, April 1996

Typeset using REVTEX 
Ultrarelativistic heavy-ion collision experiments at CERN and Brookhaven aim at the formation of quark-gluon plasma. No unambiguous signals have been detected so far, but the prospects will improve tremendously with the next generation of colliders (LHC and RHIC). Normally it is assumed that formation of a quark-gluon plasma will take place if a region in the colliding nuclei reach a combination of temperature and chemical potential that brings it into that part of the phase diagram for hadronic matter where the quark-gluon plasma has a lower free energy than a hadron gas.

Should the phase transition be of first order, the rate for quark-gluon droplet formation, $\mathcal{R}$, can be estimated in the framework of homogeneous nucleation theory [1], where

$$
\mathcal{R} \approx T^{4} \exp (-\Delta F / T) \approx 0.2 \mathrm{fm}^{-3}\left(10^{-23} \mathrm{~s}\right)^{-1} T_{100}^{4} \exp (-\Delta F / T)
$$

Here $\Delta F$ is the height of the free energy barrier which the thermal nucleation has to overcome, $T_{100}$ is the temperature in units of $100 \mathrm{MeV}$, and the rate has been expressed in units of typical heavy ion collision volumes and time scales. There is an extensive literature on the proper choice of prefactor, but since the exponential is by far the most important for our present discussion (the prefactor does not influence the relative rate suppression due to the effects discussed in our paper), we have chosen the dimensional estimate $T^{4}$. We note that a more realistic choice of prefactor [2] could change the nucleation rate by a few orders of magnitude [3]. A quantitative estimate of the effect in the present context is hindered by the fact that no derivation of the prefactor exists for a situation where curvature rather than surface tension is the most important contribution to the surface energy.

It is normally assumed that a moderate superheating is sufficient to allow droplet nucleation. This is confirmed by calculations of the droplet free energy in simple phenomenological models like, e.g., the MIT bag model [4]. Such calculations [3] have, however, neglected the requirements of color singletness and fixed momentum for the quark droplet. Both of these constraints significantly reduce the effective degrees of freedom in the quark-gluon plasma [5], thereby increasing the free energy barrier, $\Delta F$, and reducing the nucleation rate. As we show below, the nucleation rate is in fact reduced by many orders of magnitude, making 
thermal nucleation virtually impossible regardless of the amount of superheating.

We note that the ingredients of our rather simple calculation have been around in the literature for about a decade, but to our knowledge no-one has checked these dramatic consequences explicitly before.

We want to calculate the nucleation rate self-consistently within the MIT bag model, including two flavors of massless quarks, gluons, a bag pressure, volume and curvature energies, and the constraint that quark droplets must have a fixed momentum and be color neutral. It will turn out that our conclusions are not very sensitive to chemical potential, bag constant, number of quark flavors or the inclusion of the hadron gas (in most of the following we therefore just ascribe a chemical potential but no pressure to the hadrons), but very sensitive to the color singlet constraint.

Elze and Greiner [5] have derived the color singlet fixed-momentum grand canonical partition function, $Z(T, R, \mu ; p)$, for a hot, spherical quark-gluon plasma droplet of temperature $T$, radius $R$, quark chemical potential $\mu$ (corresponding to a baryon chemical potential $\left.\mu_{B}=3 \mu\right)$, and total momentum $p$. From the partition function one can find the grand potential, $\Omega=-T \ln Z$, as

$$
\Omega=T[\ln (2 \pi \sqrt{3})+4 \ln C]+1.5 T[\ln D-\ln \pi]+\frac{p^{2}}{T 4 D}+B V-T \ln Z_{0},
$$

where

$$
\begin{gathered}
\ln Z_{0} \equiv X-Y, \\
D \equiv 2 X-\frac{1}{3} Y, \\
X \equiv \frac{\pi^{2}}{12} V T^{3}\left\{12 N_{Q}\left[\frac{7}{60}+\frac{1}{2}\left(\frac{\mu}{\pi T}\right)^{2}+\frac{1}{4}\left(\frac{\mu}{\pi T}\right)^{4}\right]+\frac{32}{15}\right\}, \\
Y \equiv \frac{\pi}{18} R T\left\{12 N_{Q}\left[\frac{1}{2}+\frac{3}{2}\left(\frac{\mu}{\pi T}\right)^{2}\right]+64\right\}, \\
C \equiv \frac{1}{6} V T^{3}\left\{2 N_{Q}\left[1+3\left(\frac{\mu}{\pi T}\right)^{2}\right]+12\right\}+\frac{1}{3 \pi} R T\left(24-2 N_{Q}\right) .
\end{gathered}
$$


$N_{Q}$ is the number of massless quark flavors (we take $N_{Q}=2$ in most of the following). $V$ is the volume of the droplet. The first term in the grand potential is the contribution from the color singlet projection. The next two terms are from fixing the momentum (notice that the first of these terms survive even for $p=0$ ), and the last two terms are, respectively, the bag energy proportional to the bag constant, $B$, and the "normal" bag model grand potential of the quark-gluon gas. Notice that finite size effects, i.e. energy terms proportional to the extrinsic curvature $(8 \pi R)$ of the droplet have been included, whereas massless quarks and gluons contribute no surface tension term proportional to the bag area. One could add to Eq. (2) a surface energy from the hadron phase, as well as the hadron pressure times volume ( $\Omega \mapsto \Omega+P_{\text {Hadron }} V+\Omega_{\text {Surface,Hadron }}$, where $P_{\text {Hadron }}$ is the pressure of the hadron phase). These terms would both add positive contributions to $\Omega$, thereby further decreasing the quark-gluon droplet formation rate. For reasonable choices of parameters these terms are not decisive, and since our aim is to clearly demonstrate the enormous rate suppression due to the color singlet and momentum constraints, we have chosen not to incorporate them. Had we done that, they would only further suppress the nucleation rate and strengthen our conclusion (we return to this issue below).

The partition function was derived in a saddle-point approximation, which is expected to break down for $R T \rightarrow 0$. When $\mu=0$ it should be good to $30 \%$ for $R T=1$ and a few percent for $R T \approx 2$, and the error decreases rapidly for $\mu>0$ [5].

The grand potential as a function of droplet radius for fixed bag constant, $\mu=0$, and a temperature corresponding to nearly $30 \mathrm{MeV}$ superheating is shown in Figure 1. Curves are given with and without inclusion of the color singlet and $p=0$ constraints. One notes a significant increase in $\Omega$, in particular due to the color singlet requirement.

In Figure 2 we show the corresponding nucleation rate of quark-gluon droplets estimated according to Eq. (1). The change in free energy, $\Delta F$, necessary for formation of a critical bubble in chemical equilibrium with the surrounding hadrons at fixed temperature is just the height of the $\Omega$ barrier, so $\Delta F=\Omega$ with $\Omega$ given in Eq. (2). One recognizes the well-known reduction of the transition temperature for increased chemical potential, but what is more 
important in the present context is the significant suppression (by 4-5 orders of magnitude at extreme superheating, and much more at moderate superheating) of the nucleation rate. Units in the plot are chosen such that unity corresponds to 1 nucleation per $\mathrm{fm}^{3}$ per $10^{-23} \mathrm{~S}$ (=3 fm/c). Typical volumes and time scales relevant for quark-gluon plasma formation in ultrarelativistic heavy-ion collisions could amount to maybe $100 \mathrm{fm}^{3} \cdot \mathrm{fm} / \mathrm{c}$, so in standard calculations [3] one predicts a fair probability for bubble nucleation in the case of, say, a $10 \mathrm{MeV}$ superheating. However, the suppression resulting from requiring fixed momentum and color singletness gives a very low probability, even for (unrealistic?) superheatings of 50-100 MeV.

This surprising conclusion is not significantly influenced by changes in the assumed number of massless quarks (here taken to be 2, corresponding to infinite s-quark mass), or to the external pressure contributed by the hadron gas (the latter corresponds effectively to a small increase in $B$ [6]). Other choices of $B$ would correspond to a rescaling of $T$ and $\mu$ in Fig. 2 proportional to $B^{1 / 4}$, and of $\mathcal{R}$ proportional to $B$ (i.e., no qualitative change in the conclusion). A massive s-quark would add a surface tension term to the energy, and a surface tension contribution could come from the hadron phase as well. This would change the numbers (always in the direction of even lower nucleation probability and a slightly higher critical radius), but not the effect of color singlet suppression. No derivation exists of the color singlet partition function for a massive quark; neither does a calculation for non-zero strong coupling constant, which has therefore been assumed equal to zero in the present investigation.

The results do of course depend on the choice of model for the quark-gluon plasma. The MIT bag is certainly relevant only if a first order phase transition is involved at all. Some lattice calculations indicate, that the transition could be second order for $\mu=0$, but the issue is far from settled, and calculations for $\mu \neq 0$ do not exist. Other models would give other numerical suppression factors, but the color singlet constraint will in any case reduce the effective number of degrees of freedom [5], thereby increasing $\Delta F$ in any model, so qualitatively a rate suppression should be expected. 
Equation (2) is based on a saddle-point approximation, which is again based on an expansion of the density of states in terms of volume and curvature terms. The expansion is known to reproduce direct summation very well for $\mu=0$ [7] and for $T=0$ [8], so there is no reason to disbelieve this assumption. The uncertainty in the saddle-point approximation was discussed above. It is not negligible (but not devastating either) for critical bubbles with $R T \approx 0.9$ as typically found for superheating beyond $20 \mathrm{MeV}$ when $\mu=0$, but negligible for the much larger bubbles involved closer to the bulk phase transition temperature, and for $\mu \gg 0$ where the saddle-point approximation is much better [5]. Figure 3 shows the radius of critical bubbles as a function of temperature.

Thus, in spite of all the reservations, we conclude that a very significant suppression of the nucleation rate for quark-gluon plasma droplets in a superheated hadron gas is an inevitable consequence of the fixed momentum and color singlet constraints if the quark-hadron phase transition is first order. Other mechanisms than thermal nucleation (e.g., nucleation due to impurities) are needed to form quark-gluon plasma droplets in ultrarelativistic heavy-ion collisions if the phase transition is first order. 


\section{REFERENCES}

[1] L. D. Landau and E. M. Lifshitz, Statistical Physics (Pergamon, New York, 1980).

[2] R. Venugopalan and A. Vischer, Phys. Rev. E 49, 5849 (1994); L. P. Csernai and J. I. Kapusta, Phys. Rev. D 46, 1379 (1992).

[3] See for example J. I. Kapusta, A. P. Vischer, and R. Venugopalan, Phys. Rev. C 51, 901 (1995).

[4] T. A. DeGrand, R. L. Jaffe, K. Johnson, and J. Kiskis, Phys. Rev. D 12, 2060 (1975).

[5] H-Th. Elze and W. Greiner, Phys. Lett. B 179, 385 (1986). See also H-Th. Elze, W. Greiner, and J. Rafelski, Phys. Lett. 124B, 515 (1983); Z. Phys. C 24, 361 (1984). We note that other expressions for the color singlet partition function (without a momentum constraint) deviating from that of Elze and Greiner have been given by M. I. Gorenstein, S. I. Lipskikh, V. K. Petrov, and G. M. Zinovjev, Phys. Lett. 123B, 437 (1983), and M. G. Mustafa, Phys. Lett. B 318, 517 (1993). We have successfully reproduced the calculation of Elze and Greiner.

[6] The effect of the hadronic pressure can be calculated by substituting $B+P_{\text {Hadron }}$ instead of $B$ in Eq. (2). For $\mu=0$ an (over)estimate of $P_{\text {Hadron }}$ is the pressure of a massless pion gas with 3 degrees of freedom, $P_{\pi}=\pi^{2} T^{4} / 30$. The results with a hadron gas thus corresponds to those without a hadron gas for bag constant $B^{1 / 4}\left[1+0.33\left(T / B^{1 / 4}\right)^{4}\right]^{1 / 4}$ instead of $B^{1 / 4}$. For temperatures near the phase transition $\left(T \approx 0.7 B^{1 / 4}\right)$ this is less than a $2 \%$ effect on the scaling of $B^{1 / 4}$ and correspondingly on radii, temperatures etc. In the other extreme of very high baryon density and low $T$ the leading contribution to the hadron pressure can be approximated by a parametrisation like the Bethe-Johnson equation of state including a repulsive core via $\omega$-exchange $(\mathrm{H}$. A. Bethe and M. B. Johnson, Nucl. Phys. A 230, 1 (1974)). The simplest case for a pure neutron gas is typical for more sophisticated calculations, and gives $P_{\text {Hadron }}=364 n^{2.54} \mathrm{MeV} \mathrm{fm}^{-3}$, where $n$ is the baryon density per $\mathrm{fm}^{3}$. As for $\mu=0$ one can use the calculations without the 
hadron pressure by substituting instead of $B^{1 / 4}$ the expression $B^{1 / 4}[1+\beta]^{1 / 4}$, where $\beta=$ $0.017\left((200 \mathrm{MeV})^{4} / B\right)\left(n / n_{\text {nuc }}\right)^{2.54}$, and $n_{\text {nuc }}$ is nuclear matter density. The corresponding changes in radii etc are small unless one goes to very extreme densities $\left(0.4 \%\right.$ for $n=n_{\text {nuc }}$ and $19 \%$ for $n=5 n_{\text {nuc }}$ respectively). In any case, a hadron pressure always further suppresses the rate for thermal nucleation at fixed $T, \mu$.

[7] I. Mardor and B. Svetitsky, Phys. Rev. D 44, 878 (1991).

[8] J. Madsen, Phys. Rev. D 50, 3328 (1994). 


\section{FIGURES}

FIG. 1. The grand potential, $\Omega$, as a function of droplet radius for $B^{1 / 4}=200 \mathrm{MeV}, \mu=0$, and $T=170 \mathrm{MeV}$. The lower curve is without momentum and color singlet constraints, middle curve with the color singlet constraint, and upper curve with both constraints included (at zero momentum).

FIG. 2. The nucleation rate of quark-gluon droplets as a function of temperature for $B^{1 / 4}=200 \mathrm{MeV}$. Solid curves for quark chemical potential zero, dotted curves for $\mu=100 \mathrm{MeV}$, dashed curves for $\mu=300 \mathrm{MeV}$, dot-dash curves for $\mu=400 \mathrm{MeV}$, and dash-triple dot curves for $\mu=500 \mathrm{MeV}$. The lower set of curves includes the color and momentum constraints; the upper set does not.

FIG. 3. Radius of critical bubbles as a function of temperature for calculations including color and momentum constraints, for chemical potentials as in Fig. 2. 


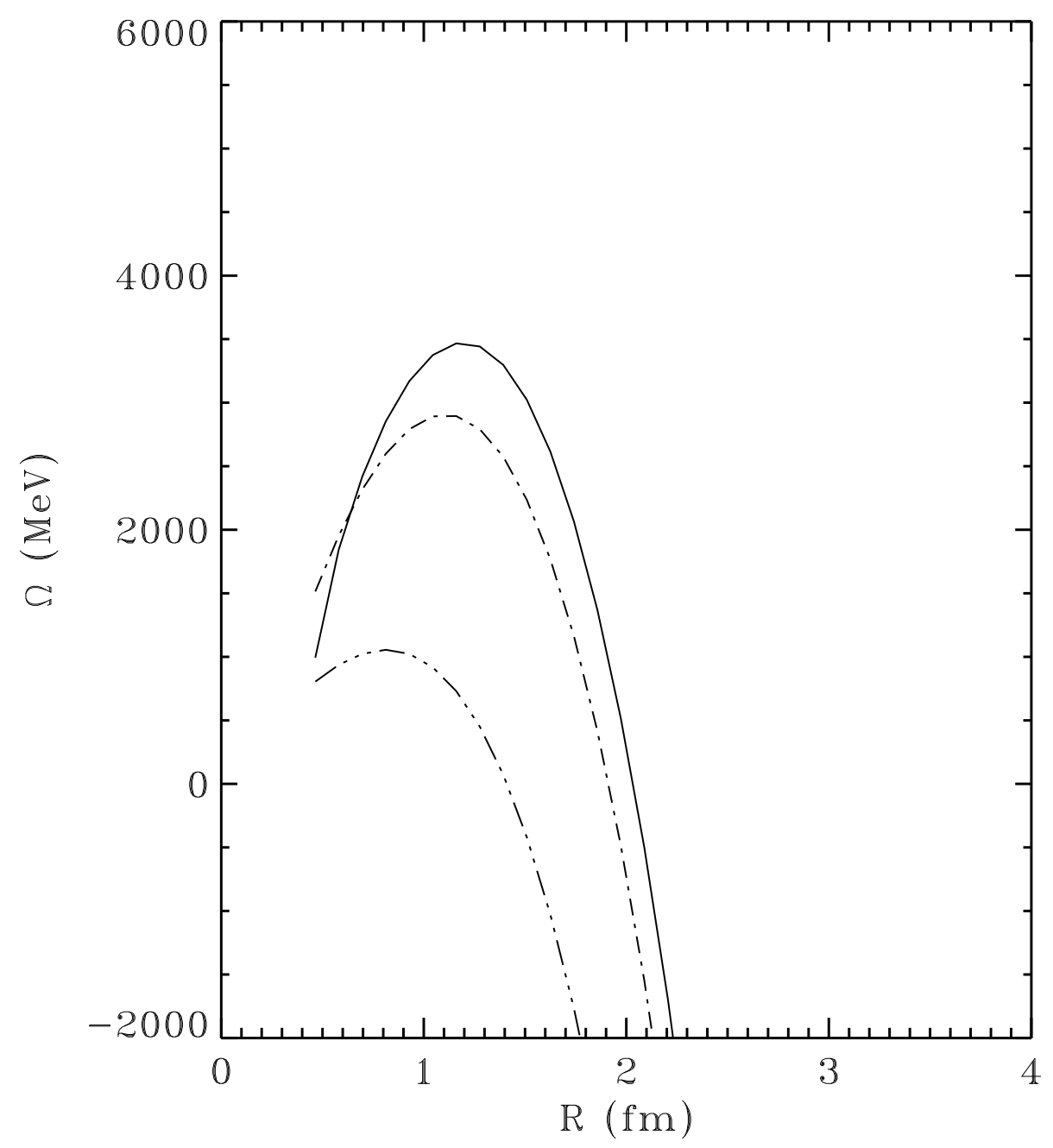




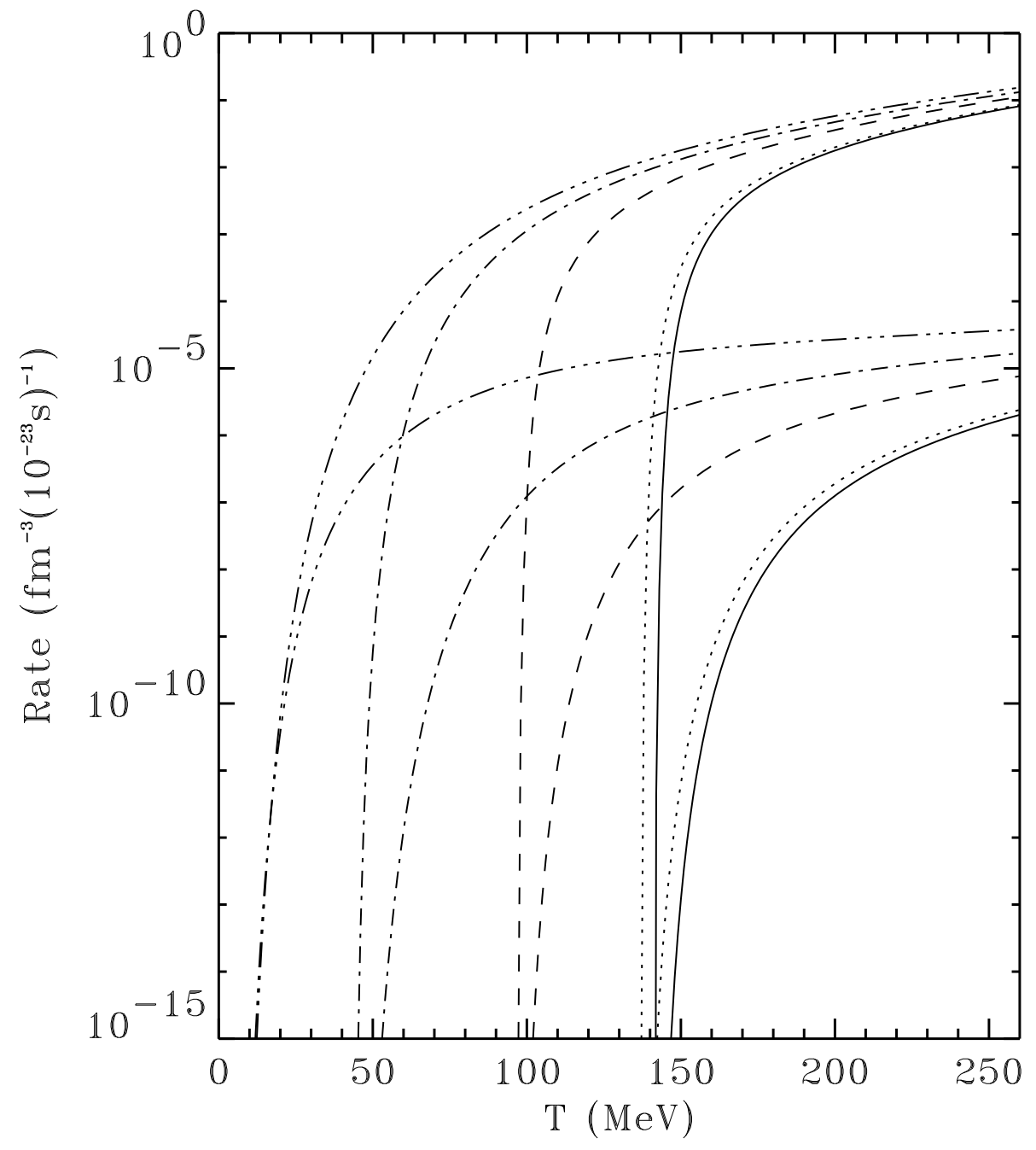




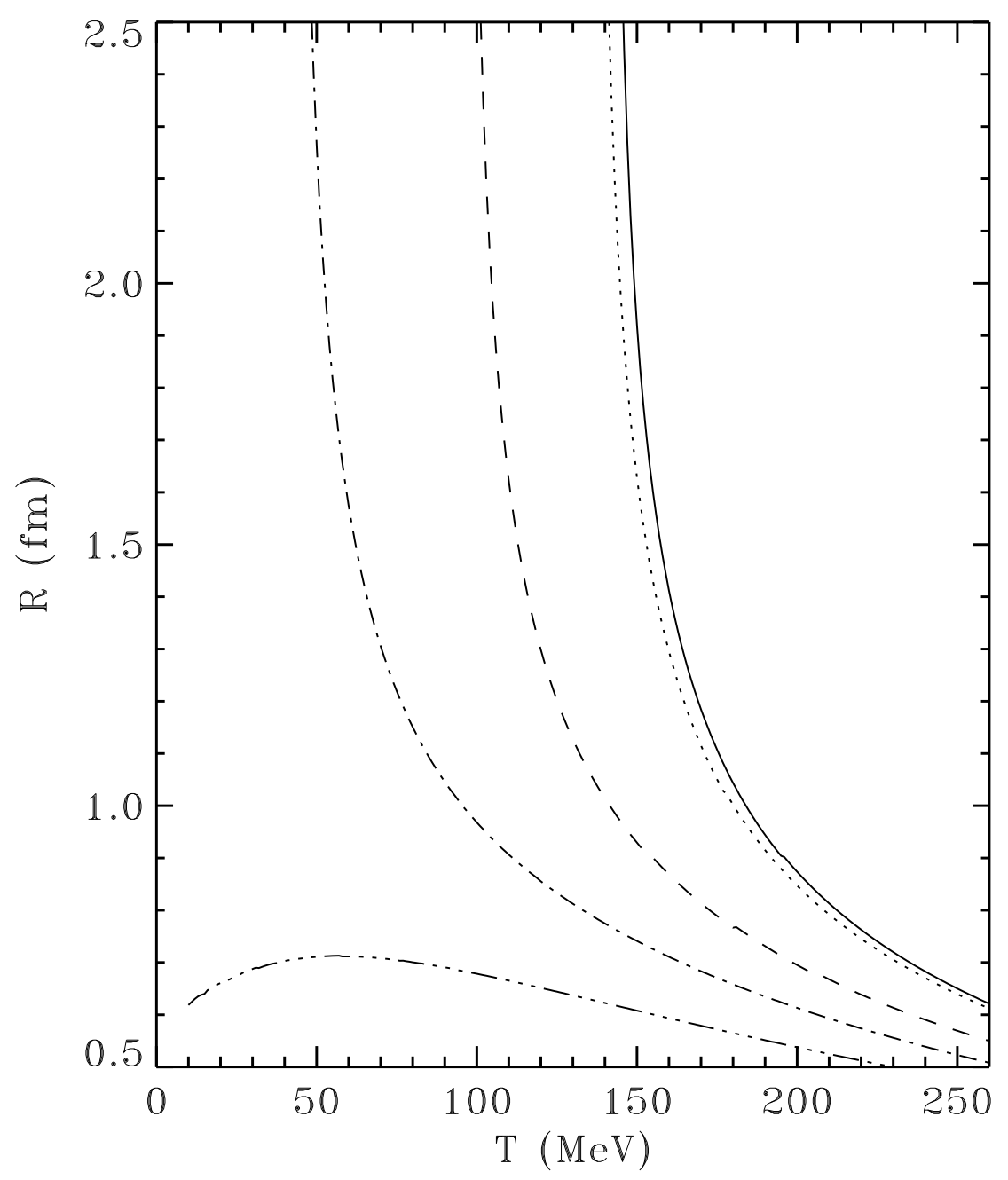

